\title{
Micromegas at low pressure for beam tracking
}

\author{
J. Pancin, ${ }^{a}$ B. Fernández, ${ }^{b, 1}$ S. Damoy, ${ }^{a}$ M. Kebbiri, ${ }^{c}$ T. Papaevangelou ${ }^{c}$ and \\ M. Riallot ${ }^{c}$ \\ ${ }^{a}$ GANIL, CEA/DSM-CNRS/IN2P3, \\ Bvd H. Becquerel, Caen, France \\ ${ }^{b}$ CNA, University of Seville, \\ C/Thomas A. Edison, Seville, Spain \\ ${ }^{c} C E A-D S M / I R F U$, \\ Gif Sur Yvette, France \\ E-mail: bfernandous.es
}

ABSTRACT: New facilities like FAIR at GSI or SPIRAL2 at GANIL, will provide radioactive ion beams at low energies (less than $10 \mathrm{MeV} / \mathrm{n}$ ). Such beams have generally a large emittance, which requires the use of beam tracking detectors to reconstruct the exact trajectories of the nuclei. To avoid the angular and energy straggling that classical beam tracking detectors would generate in the beam due to their thickness, we propose the use of SED (Secondary Electron Detectors). It consists of a low pressure gaseous detector placed outside the beam coupled to an emissive foil in the beam. Since 2008, different low pressure gaseous detectors (wire chambers and micromegas) have been constructed and tested. The performances achievable at low pressure are similar to or even better than the ones at atmospheric pressure. The fast charge collection leads to excellent timing properties as well as high counting rate capabilities. Several micromegas at low pressure were tested in the laboratory demonstrating a good time resolution, $130 \pm 30 \mathrm{ps}$, which is compatible with the results obtained with wire chambers.

KEYWORDS: Micropattern gaseous detectors (MSGC, GEM, THGEM, RETHGEM, MHSP, MICROPIC, MICROMEGAS, InGrid, etc); Particle tracking detectors (Gaseous detectors)

\footnotetext{
${ }^{1}$ Corresponding author.
} 


\section{Contents}

1 Introduction 1

2 SED principle 2

3 The micromegas prototype 2

4 Time resolution measurements $\quad 4$

5 Conclusion $\quad 7$

\section{Introduction}

FAIR at GSI (Darmstadt, Germany) and SPIRAL2 at GANIL (Caen, France) are new accelerators in construction. These new facilities will be able to produce high intensity, up to $10^{6} \mathrm{pps} / \mathrm{cm}^{2}$, and low energy, less than $10 \mathrm{MeV} / \mathrm{n}$, Radioactive Ion Beams (RIBs). Due to the characteristics of these ions beams, they normally have large emittance. It is necessary to use Beam Tracking Detectors (BTD) to reconstruct the exact trajectories of nuclei in order to know for example, the kinematics of the nuclear reactions and to study the structure of exotic nuclei. BTD that are currently used in GANIL, such as CATS (Chambre à Trajectoire de Saclay) [1] are not suitable for the new RIBs because of their thickness $\left(\sim 600 \mu \mathrm{g} / \mathrm{cm}^{2}\right)$. They induce too much energy and angular straggling on low energy beams. With the aim of minimizing the material thickness in the beam and to improve the counting rate capabilities of BTD, a study based on SED (Secondary Electron Detectors) [2], was initiated by IRFU (CEA-Saclay) [3]. It consists of a thin foil ( $\sim 180 \mu \mathrm{g} / \mathrm{cm}^{2}$ at $45^{\circ}$ ) of aluminized mylar in the beam coupled to a low pressure gaseous detector outside the beam.

Since 2008, different prototypes of low pressure gaseous detectors (wire chambers and for the first time, one micromegas at low pressure) have been constructed and tested in the laboratory [3] with a fission fragment source $\left({ }^{252} \mathrm{Cf}\right)$. These prototypes were tested in the beam $\left({ }^{82} \mathrm{Kr}^{+10}\right.$ ion beam at $1.7 \mathrm{MeV} / \mathrm{n}$ ) at GANIL in 2010 [4]. The results obtained in terms of time and spatial resolution and counting rate capabilities were promising of course for the wire chambers but also for the micromegas at low pressure. Following these promising results, and having in mind the objective of reaching a counting rate of $10^{6} \mathrm{pps}$ without degrading the good time resolution obtained previously in the beam $(175 \pm 30 \mathrm{ps})$, we have decided to perform a complete study using different geometries for the micromegas at low pressure. Several micromegas with different amplification gaps have been constructed.

In this article, the different micromegas prototypes are described and the set-up used for time resolution measurements in the laboratory is presented. In addition, we show the study performed in terms of gain capabilities and time resolution depending on the geometry of the micromegas. 


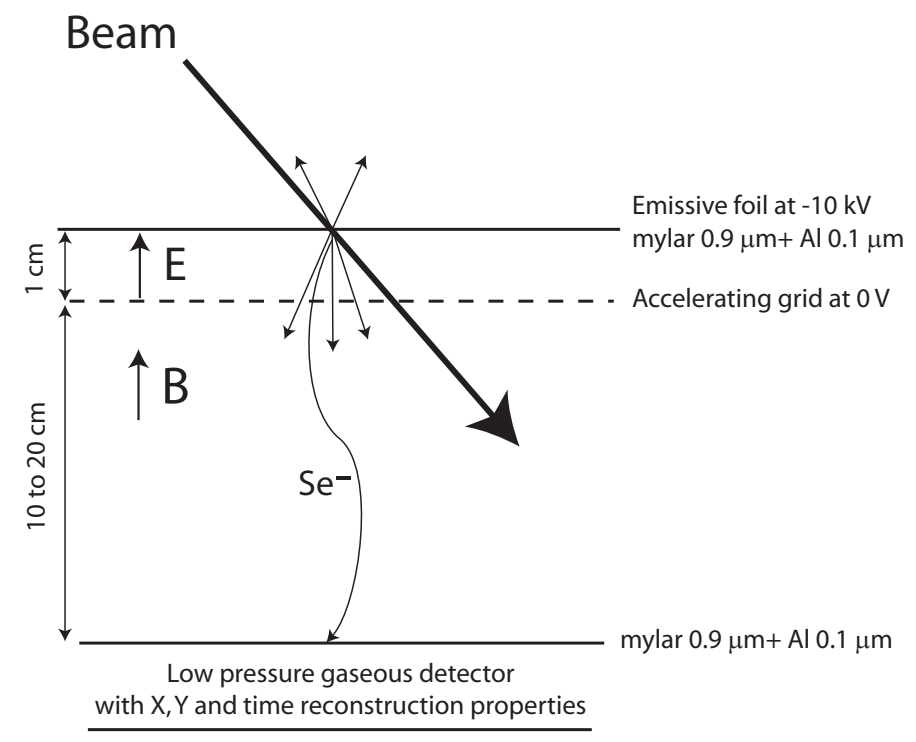

Figure 1. Schematic of the SED principle.

\section{SED principle}

The principle of operation of the Secondary Electron Detectors at low pressure is described in details in [2] and shown on figure 1. A thin emissive foil, made out of $0.9 \mu \mathrm{m}$ thick aluminized mylar is placed in the beam at $45^{\circ}$ with respect to the beam line. A voltage of $-10 \mathrm{kV}$ is applied on it and a grounded grid is positioned at $10 \mathrm{~mm}$ from the foil. When the beam passes through the emissive foil some electrons are ejected from the surface of the foil. Typically, fission fragments at $1 \mathrm{MeV} / \mathrm{n}$ will create 100 to 200 secondary electrons. Thanks to the electric field resulting from the high voltage applied on the foil, these electrons are accelerated, with a mean energy of $10 \mathrm{keV}$, towards the entrance window of the SED. This detector is placed outside the beam at several centimeters from the emissive foil. Due to the distance between the emissive foil and the SED, a magnetic field is necessary if a good spatial resolution is required. The secondary electrons $\left(\mathrm{Se}^{-}\right)$ have a transverse velocity component leading to deviations of several millimeters between their emission point on the emissive foil and the entrance window of the detector. Thanks to the magnetic field, they travel in spirals and get focused [5]. After crossing the entrance window of the detector with $70 \%$ efficiency, they ionize the gas. The gas usually used at low pressure is pure isobutane for its low ionization energy and its high quenching power. The energy deposit per electron is about $100 \mathrm{eV}$ in $2 \mathrm{~mm}$ of isobutane at 4 torr which gives around 4 ionization electrons per secondary electron. The ionization electrons are then multiplied by an avalanche process generally in wire chambers. The signal generated is fast since the electron drift velocity is higher than $10 \mathrm{~cm} / \mu \mathrm{s}$ for an electric field higher than $1 \mathrm{kV} / \mathrm{cm}$.

\section{The micromegas prototype}

Micromegas detectors have been invented in 1996 and they are now widely used in particle or nuclear physics $[6,7]$. The advantages compared to wire chambers have been demonstrated several 


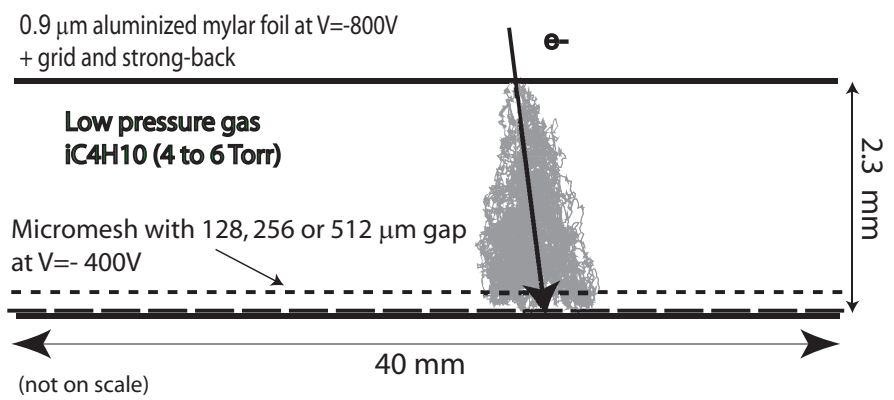

Figure 2. Schematic of the micromegas detector.

times but more at normal pressure than at low pressure. The purpose of this study is to check the functioning of this detector at low pressure (several torr) and to compare its performances to classical wire chambers. Wire chambers are known to work very well at low pressure [8].

Micromegas detectors are composed of two zones separated by a micromesh, a drift gap of several millimeters with a weak electric field and an amplification gap of several tens of microns with a strong electric field. When a charged particle passes through the gaseous detector, ionization electrons are created mainly in the drift gap. They drift towards the micromesh, and are transferred to the amplification gap where they get multiplied by an avalanche process. A signal can thus be observed either on the micromesh or on some strips facing the mesh on the opposite side of the amplification gap. However, the working points are different at low pressure.

The reduced critical electric field $E_{c} / P$ in $\mathrm{V} \cdot \mathrm{cm}^{-1} \cdot \mathrm{torr}^{-1}$ is a characteristic value of the gas at which an avalanche begins (between 40 and $90 \mathrm{~V} \cdot \mathrm{cm}^{-1} \cdot \mathrm{torr}^{-1}$ for most gases) [9]. A field of only $400 \mathrm{~V} / \mathrm{cm}$ is enough to initiate the avalanche process at a low pressure of 4 torr. It is easy for example to induce amplification in the drift gap. However, the mean free path of the ionization electrons under the electric field is high at low pressure (up to several tens of microns). The amplification gaps of $100 \mu \mathrm{m}$ usually used in micromegas detectors are hence too small to get enough signal. There are then two ways to do amplification using a micromegas detector at these pressures. It is possible either to use a large amplification gap [10] (more than 500 microns) or to do a pre-amplification in the drift gap keeping a small amplification gap. The first solution is preferable to keep high count-rate capabilities but it means the amplification gap has to be increased.

Three new prototypes of micromegas detectors have been constructed, one with a $128 \mu \mathrm{m}$ thick amplification gap, one with $256 \mu \mathrm{m}$ and the last detector with $512 \mu \mathrm{m}$ (see figure 2). All of them consist of a 1D strip plane of $1.27 \mathrm{~mm}$ strip pitch and $4 \times 4 \mathrm{~cm}^{2}$ area on which some pillars are deposited by a bulk process [11]. The size of the amplification gap is changed thanks to the numbers of photoresist layers laminated onto the strip plane. A $5 \mu \mathrm{m}$ thick nickel micromesh is then deposited on the pillars. The drift gap is $2.3 \mathrm{~mm}$ high, minus the amplification gap. The entry mylar foil of $0.9 \mu \mathrm{m}$ thickness is also the drift electrode. The homogeneity of the drift gap is ensured by putting a strong back on the entry foil to avoid any deformation due to the gas pressure. These detectors have been tested in terms of signal amplitude and also time resolution. 


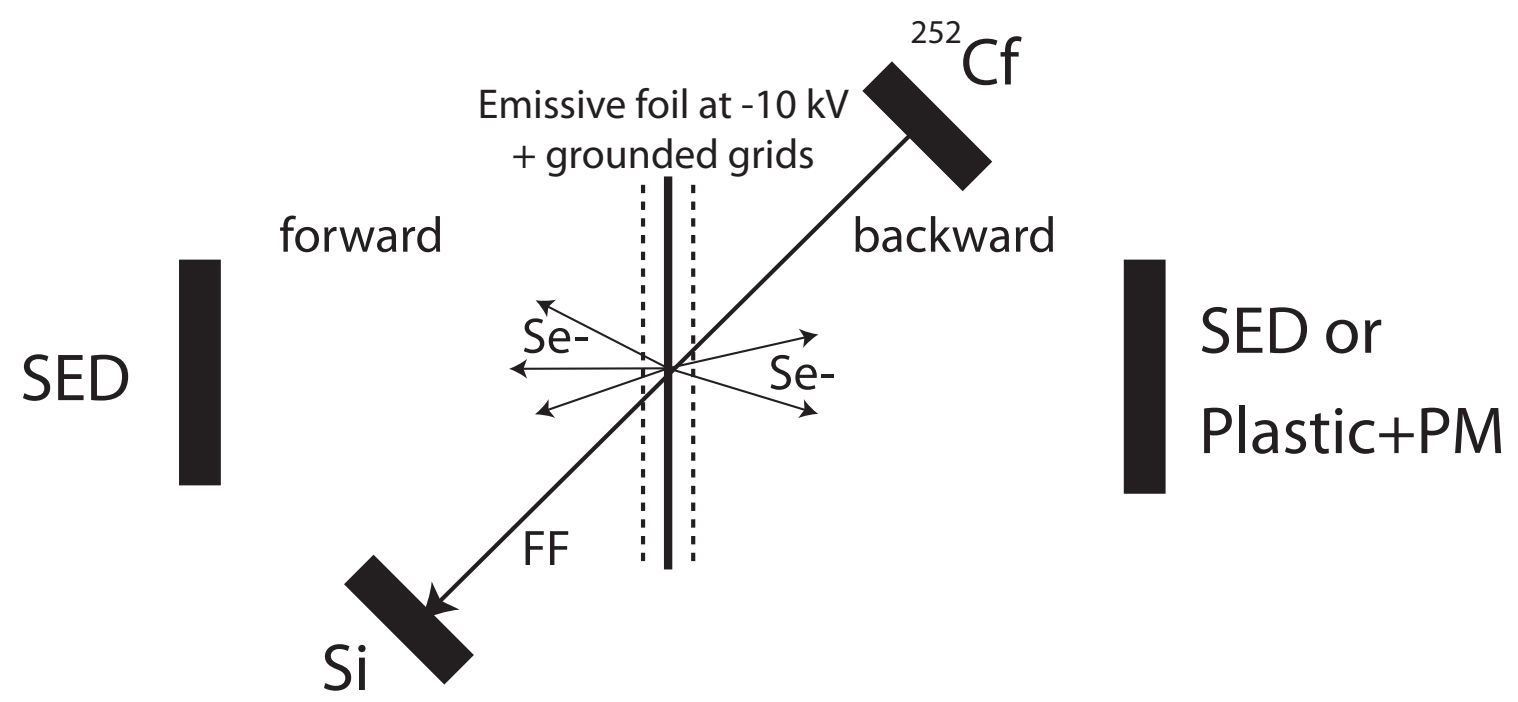

Figure 3. Experimental set-up for the time resolution measurements in the laboratory.

\section{Time resolution measurements}

The time resolution is an important issue for these detectors. This measurement is generally performed using a ${ }^{252} \mathrm{Cf}$ source. The figure 3 shows the experimental set up used in the laboratory to measure the time resolution. The source is placed at $45^{\circ}$ from a square emissive foil $\left(70 \times 70 \mathrm{~mm}^{2}\right)$ polarized at $-10 \mathrm{kV}$ with a grounded extraction grid on each side. These grids are used to extract the secondary electrons created by the fission fragments but also to give them enough energy to pass the entry foil of the SED. A silicon detector is placed in front of the source on the other side from the emissive foil. It is used to trigger the acquisition system on the fission fragments, preferentially on the light fission fragments since they are the fastest. The micromegas detectors have been tested one by one in front of the emissive foil in the forward direction of the fission fragments (on the silicon detector side). A second detector (either a second low pressure detector or a fast plastic scintillator equipped with a photomultiplier) is placed in front of the emissive foil but backward in order to have three detectors in the system. The acquisition system consists mainly of a matacq card [12] permitting to register the signal of each detector. The analysis is done off-line by applying a software CFD (Constant Fraction Discriminator) to the signals to get the start time of each detector [3]. The detector to be tested is connected to a fast amplifier (rise time below $2 \mathrm{~ns}$ and amplification factor of 200). The mean amplitudes that we have measured with the different micromegas detectors were usually higher than $200 \mathrm{mV}$ with a noise of about $20 \mathrm{mV}$ peak to peak, a mean total rise time below $10 \mathrm{~ns}$ and an overall time length of $60 \mathrm{~ns}$. The time difference distributions of each couple of detectors permit to estimate the time resolution of the three detectors. When the fast plastic scintillator is used and since it has a time resolution close to $30 \mathrm{ps}$, the time difference between the plastic scintillator and the forward SED detector gives directly the time resolution of the forward detector. Although this method gives an upper limit of the time resolution of the micromegas detector, it is independent of the velocity distribution of the fission fragments and is more accurate with low error bars. 


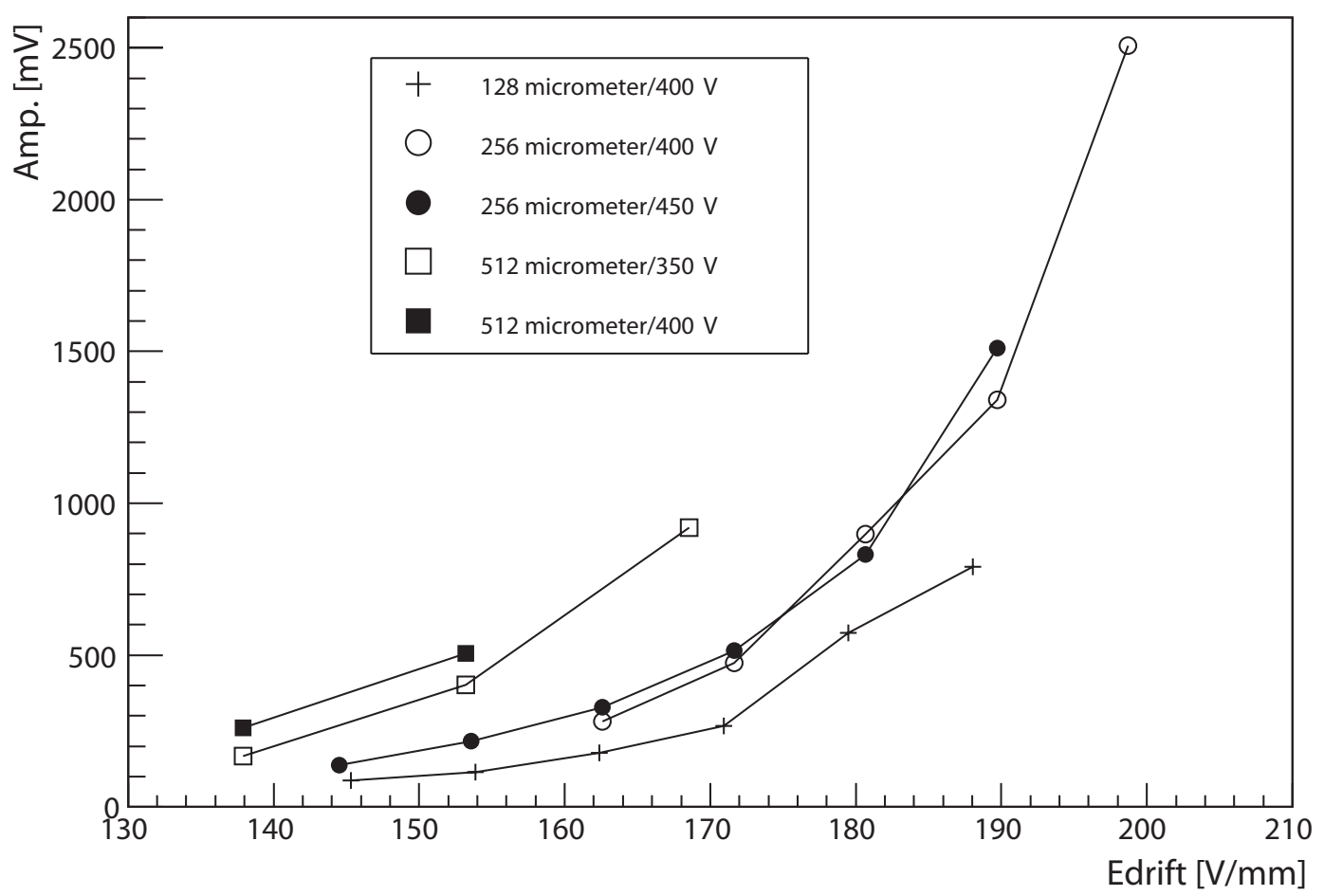

Figure 4. Mean amplitude of the signals at 4 torr for the three prototypes versus the electric field in the drift gap for different amplification voltages.

The figure 4 shows the mean amplitude signals at 4 torr of the three micromegas detectors versus the electric field in the drift gap for different voltages in the amplification gap. The first conclusion is the very good signal over noise ratio of the micromegas compared to a classical wire chamber used as SED or to the previous micromegas we had realized [3]. The mean amplitude can go up to $2500 \mathrm{mV}$ at $200 \mathrm{~V} / \mathrm{mm}$, which is at least a factor of 10 higher than with a wire chamber in the same conditions (same pressure and maximum voltage). It should permit to lower the threshold of detection and to improve the detection efficiency of low $Z$ particles passing through the emissive foil. The use of the entry foil as the drift electrode permits to gather much more of the secondary electrons and hence to improve the signal amplitude. This figure also shows that the signal increases with the size of the amplification gap, although the amplification electric field decreases. Since the townsend coefficient $(\alpha)$ gets saturated at high electric field and low pressure [9], the gain is more important only thanks to the increase of the gap. It confirms that our detector works correctly in micromegas mode at low pressure. The fact that the signal amplitude remains the same for two different amplification voltages in the micromegas with the $256 \mu \mathrm{m}$ amplification gap, is another indication of the saturation of $\alpha$. It is not the case for the detector with the $512 \mu \mathrm{m}$ gap where the amplification electric field becomes smaller and $\alpha$ is not constant anymore. Unfortunately, it was not possible to increase the amplification voltage while increasing the amplification gap. The sparking limit was the same (around $-400 \mathrm{~V}$ ) whatever was the amplification gap but this problem is not fully understood yet.

The figure 5 shows the time resolution at 4 torr of the three micromegas detectors versus the electric field in the drift gap for different voltages in the amplification gap. The resolution has been 


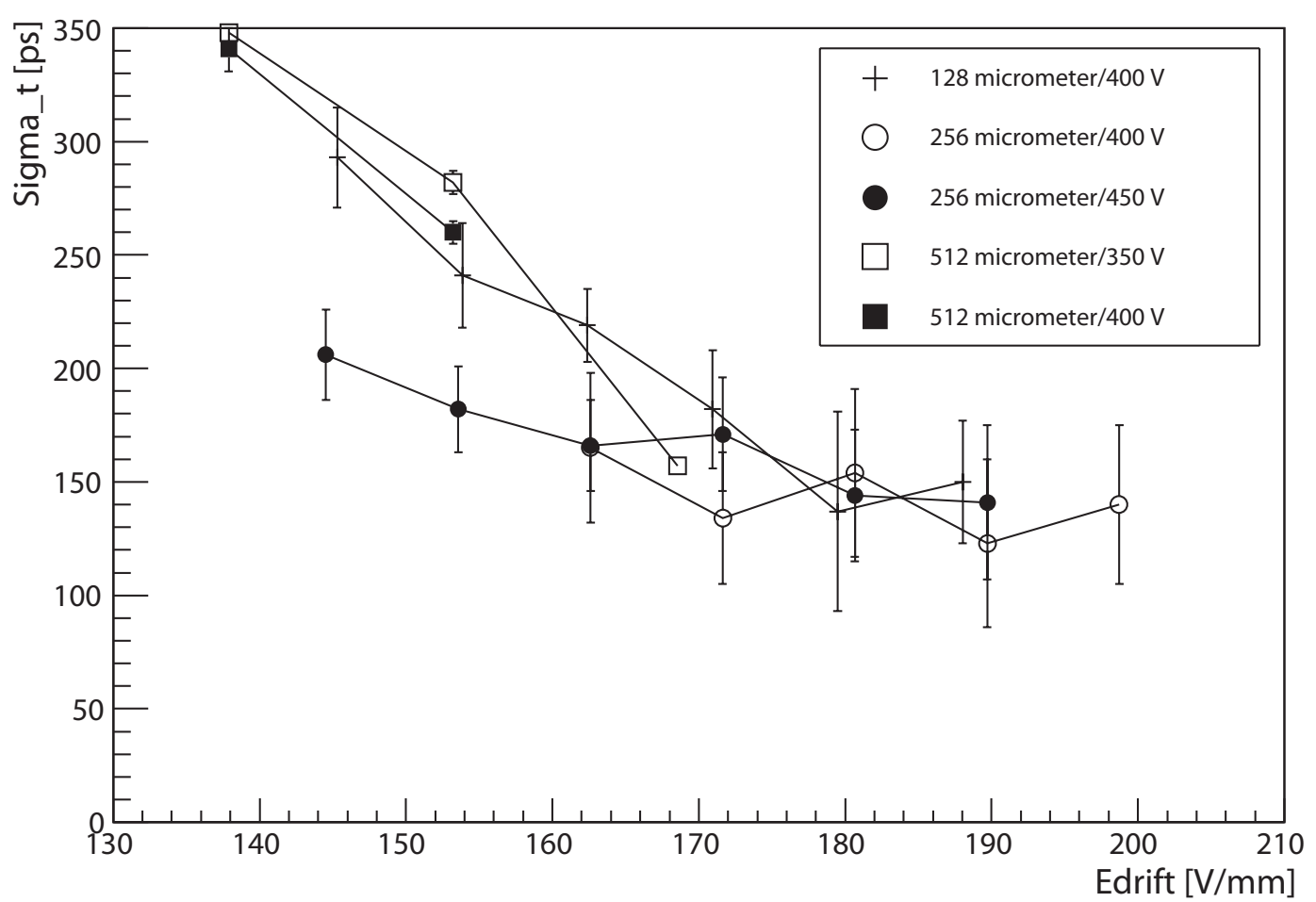

Figure 5. Time resolution at 4 torr for the three prototypes versus the electric field in the drift gap for different amplification voltages.

improved largely compared to the last paper [3] with a result close to $130 \mathrm{ps}$. We are approaching the time resolution of our wire chambers which is about 120 ps at $200 \mathrm{~V} / \mathrm{mm}$ (electric field far from the wires in the so-called PPAC region). Thanks to the new design, the amplification and the drift gaps are more homogeneous in thickness. The fact to have increased the signal amplitude is also responsible for this time resolution improvement. The error bars quoted in the case of the detector having a $512 \mu \mathrm{m}$ amplification gap, are smaller since the plastic scintillator was used for these measurements. This figure seems to indicate that the time resolution does not depend on the amplification gap but more on the drift field, like in a parallel plate chamber. The time resolution is hence more influenced by the electrons created in the drift gap. A high drift field and as a consequence a pre-amplification in the drift gap is necessary to get a good time resolution. The fast amplifier permits to follow the time evolution of the signal. Once the ionization electrons have been created mainly in the drift gap, they begin to drift and to be multiplied. The signal is observed as soon as the electrons have crossed the micromesh and their number is high enough to have a good signal over noise ratio. It means that the beginning of the signal, which corresponds to low CFD values, is induced by the electrons that have been created in the drift gap closer to the micromesh. It is hence not necessary to wait for all the electrons drifting in the $2 \mathrm{~mm}$ height drift gap before to see a signal and to get a good time information. The time resolution of the detector having a $256 \mu \mathrm{m}$ amplification gap, is interesting since it depends less on the drift field than for the detector with the $128 \mu \mathrm{m}$ amplification gap. The micromegas with the $512 \mu \mathrm{m}$ amplification gap has a strange behavior probably due to the low micromesh sparking limit. Its behavior should look more like the 
detector that has a $256 \mu \mathrm{m}$ amplification gap. This low sparking limit does not allow this detector to be operated with less pre-amplification in the drift gap, which was the purpose of using this high amplification gap micromegas $(512 \mu \mathrm{m})$ for high count-rate applications. However, these results demonstrate that a high drift field is necessary to achieve both a good signal over noise ratio and the best time resolution.

\section{Conclusion}

A strong improvement has been achieved in terms of time resolution with micromegas detectors at low pressure (around 4 torr). A resolution of $130 \pm 30$ ps has been measured for several detector geometries which is quite comparable to the resolution of a wire chamber of equivalent gap. It opens the possibility to use this detector at low pressure for beam tracking applications with low energy ions (from 2 to $10 \mathrm{~A} . \mathrm{MeV}$ ). However, the performances of these detectors have still to be measured in the beam and to be compared to a wire chamber. A new detector of large size $\left(20 \times 15 \mathrm{~cm}^{2}\right.$ area) will be constructed in 2012 to realize spatial resolution measurements and tests in the beam.

\section{Acknowledgments}

We would like to thank the people at CEA-Saclay from IRFU/SEDI, who work in the bulk laboratory, for constructing the micromegas bulk detectors. This work is supported by the Spanish Research Project FPA2009-08848.

\section{References}

[1] S. Ottini-Hustache et al., CATS, a low pressure multiwire proportional chamber for secondary beam tracking at GANIL, Nucl. Instrum. Meth. A 431 (1999) 476.

[2] A. Drouart et al., Very large emissive foil detectors for the tracking of low-energy heavy ions, Nucl. Instrum. Meth. A 579 (2007) 1090.

[3] J. Pancin et al., Secondary electrons detectors for beam tracking: micromegas and wire chamber, 2009 JINST 4 P12012.

[4] M.A.G. Alvarez et al., Results of the in-beam tests of secondary electrons detectors on CIME at GANIL, GANIL Internal Report/STP 226 (2010).

[5] O.H. Odland et al., A fast position sensitive microchannel plate detector for ray-tracing of charged particles, Nucl. Instrum. Meth. A 378 (1996) 149.

[6] I. Giomataris, Development and prospect of the new gaseous detector “Micromegas”, Nucl. Instrum. Meth. A 419 (1998) 239.

[7] J. Derré and I. Giomataris, Recent experimental results with MICROMEGAS, Nucl. Instrum. Meth. A 477 (2002) 23.

[8] A. Breskin, R. Chechik and N. Zwang, Heavy ion timing with very low pressure MWPCs, Nucl. Instrum. Meth. 165 (1979) 125. 
[9] Yu.I. Davydov, On the first Townsend coefficient at high electric field, IEEE Trans. Nucl. Sci. 53 (2006) 2931 [physics/0409156].

[10] M. Nakhostin, Performance of a low-pressure Micromegas-like gaseous detector, Nucl. Instrum. Meth. A 598 (2009) 496.

[11] I. Giomataris et al., Micromegas in a bulk, Nucl. Instrum. Meth. A 560 (2006) 405 [physics/0501003].

[12] D. Breton, E. Delagnes and M. Houry, Very high dynamic range and high sampling rate VME digitizing boards for physics experiments, IEEE Trans. Nucl. Sci. 52 (2005) 2853. 